\title{
Novel Subclone of Carbapenem-Resistant Klebsiella pneumoniae Sequence Type 11 with Enhanced Virulence and Transmissibility, China
}

\author{
Kai Zhou, ${ }^{1}$ Tingting Xiao, ${ }^{1}$ Sophia David, ${ }^{1}$ Qin Wang, Yanzi Zhou, Lihua Guo, David Aanensen, \\ Kathryn E. Holt, Nicholas R. Thomson, Hajo Grundmann, ${ }^{2}$ Ping Shen, ${ }^{2}$ Yonghong Xiao ${ }^{2}$
}

\begin{abstract}
We aimed to clarify the epidemiologic and clinical importance of evolutionary events that occurred in carbapenem-resistant Klebsiella pneumoniae (CRKP). We collected 203 CRKP causing bloodstream infections in a tertiary hospital in China during 2013-2017. We detected a subclonal shift in the dominant clone sequence type (ST) 11 CRKP in which the previously prevalent capsular loci (KL) 47 had been replaced by KL64 since 2016. Patients infected with ST11-KL64 CRKP had a significantly higher 30-day mortality rate than other CRKP-infected patients. Enhanced virulence was further evidenced by phenotypic tests. Phylogenetic reconstruction demonstrated that ST11-KL64 is derived from an ST11-KL47-like ancestor through recombination. We identified a pLVPK-like virulence plasmid carrying rmpA and peg-344 in ST11-KL64 exclusively from 2016 onward. The pLVPK-like-positive ST11-KL64 isolates exhibited enhanced environmental survival. Retrospective screening of a national collection identified ST11-KL64 in multiple regions. Targeted surveillance of this high-risk CRKP clone is urgently needed.
\end{abstract}

$\mathrm{T}$ he global dissemination of carbapenem-resistant Enterobacteriaceae (CRE) has become an urgent public health concern $(1,2)$. In 2016, the World Health

Author affiliations: First Affiliated Hospital of Southern University of Science and Technology (Shenzhen People's Hospital),

Shenzhen, China (K. Zhou); The Second Clinical Medical College of Jinan University, Shenzhen (K. Zhou); Zhejiang University, Hangzhou, China (T. Xiao, Q. Wang, Y. Zhou, L. Guo, P. Shen, Y. Xiao); Centre for Genomic Pathogen Surveillance, Cambridge, UK (S. David, D. Aanensen); University of Melbourne, Melbourne, Victoria, Australia (K.E. Holt); London School of Hygiene and Tropical Medicine, London, UK (K.E. Holt, N.R. Thomson); Wellcome Trust Sanger Centre, Cambridge (N.R. Thomson); University of Freiburg, Freiburg, Germany (H. Grundmann)
Organization included CRE in a list of antimicrobial-resistant priority pathogens on which to concentrate future drug development strategies. Of note, carbapenem-resistant Klebsiella pneumoniae (CRKP) account for $60 \%-90 \%$ of clinical CRE infections in the United States, Europe, and China (1-3), resulting in an increased mortality rate of up to $40 \%-50 \%$ in nosocomial settings (4).

The dissemination of CRKP is mostly clonal, and the population structure is geographically specific. Since its emergence during the early to mid-2000s, sequence type (ST) 258 has become the most prevalent CRKP clone in North America, Latin America, and Europe (5). However, in Asia, especially China, ST11 is the predominant clone, accounting for up to $60 \%$ of CRKP (3). ST11 is a single-locus (tonB) variant of ST258, and both types belong to the clonal group 258. A recombination event is thought to have occurred between a recipient ST11 and a donor ST442like strain, giving rise to ST258 during 1985-1997 $(6,7)$. A phylogenomic study revealed that the ST258 population consists of $>2$ clades, resulting from an $\approx 215-\mathrm{kb}$ recombination event that includes the capsule polysaccharide (cps) synthesis locus (6). The genetic differences generated by the resulting capsular switch are supposed to be primarily responsible for the ST258 diversification (8). Likewise, a segregation was identified in the ST11 population, resulting in $>3$ clades with different capsular loci (KL) (9-11). These studies consistently indicate that cps is a recombination hotspot in K. pneumoniae. However, the K-type distribution within ST11 in clinical settings is unclear. More important, the biological, epidemiologic, and

${ }^{1}$ These first authors contributed equally to this article.

${ }^{2}$ These senior authors contributed equally to this article. 
clinical importance of capsular switches in ST11 remains poorly understood.

Of greater concern, a carbapenem-resistant hypervirulent $K$. pneumoniae ST11 outbreak clone was recently reported in eastern China (12). The outbreak strain was KL47 and hypermucoid and harbored a virulence plasmid carrying rmpA2 and the aerobactin synthesis locus. Loss of the plasmid substantially alleviated virulence in a Galleria mellonella moth model. This finding indicates a worrying convergence of carbapenem resistance and hypervirulence in an already epidemic lineage of $K$. pneumoniae. Although incidence of carbapenem-resistant hypervirulent $K$. pneumoniae has remained low (13-15), understanding how this lineage emerged and evolved is crucial in controlling its further dissemination.

In this study, we measured the occurrence and clinical outcomes of bloodstream infections (BSI) caused by CRKP in a tertiary hospital in China during 2013-2017. We characterized the genomic alterations in the dominant ST11 population and ascertained associated changes in phenotype and pathogenicity traits.

\section{Materials and Methods}

\section{Setting and Study Design}

We performed a retrospective study in a 2,500-bed tertiary care hospital in China during January 2013-June 2017. We reviewed medical records of any patient with a blood culture positive for K. pneumoniae and a clinical course consistent with bacteremia (upon notification of the patient). Patients $<16$ years of age were excluded. If 1 patient had $>1$ episode of BSI caused by K. pneumoniae (BSI-KP), we included only the first episode. This study was approved by the institutional review board of the First Affiliated Hospital of Zhejiang University in China (approval no. 2017-442). Definitions of terms are detailed in Appendix 1 (https:/ / wwwnc.cdc.gov/ EID/article/26/2/19-0594-App1.pdf).

\section{Microbiological Assessment}

We determined antimicrobial susceptibility by using the VITEK-II system (bioMérieux, https:/ / www. biomerieux.com) and further confirmed by using the broth microdilution method. We defined carbapenem nonsusceptibility as MIC $>2 \mathrm{mg} / \mathrm{L}$ for imipenem or meropenem or MIC $>1 \mathrm{mg} / \mathrm{L}$ for ertapenem (16). We used multilocus sequence typing to identify ST11 (17). We estimated the pathogenicity of K. pneumoniae by testing $G$. mellonella infection, biofilm production, and neutrophil-killing resistance, as previously described (18-20) (Appendix 1). We evaluated the capacity of CRKP to survive on dry surfaces over time by using previously described methods (21), except that the stainless steel discs were replaced by Corning 24-well polystyrene microplates (Merck, https:/ / www.sigmaaldrich.com) and the concentration of bacteria was adjusted to $1 \times 10_{8} \mathrm{CFU} / \mathrm{mL}$.

\section{Whole-Genome Sequencing and Analyses}

We sequenced 154 ST11 isolates by using an Illumina Hiseq2500 instrument (Illumina, https://www.illumina.com) with $2 \times 125$-bp paired-end libraries. We performed de novo assembly of the short-read data by using CLC Genomics Workbench version 10.0 (QIAGEN, https://www.qiagen.com) after quality trimming (Phred quality score $>20$ ). We performed longread sequencing on 2 isolates (KP16932 and KP47434) by using the PacBio RSII platform (Pacific Biosciences, https://www.pacb.com) with a 10-kb library. A hybrid assembly of these 2 isolates was generated by using Unicycler 0.4.0 (22) with the short and long reads. We annotated the assemblies by using the RAST server (https:/ / rast.nmpdr.org) and conducted multilocus sequence typing by using the CGE server (https:/ / cge. cbs.dtu.dk). We performed plasmid analysis by Southern blotting and Blast (Appendix 1). We determined the presence or absence of resistance and virulence genes by using Ariba (23) with a custom gene database (https:/ / figshare.com/s/94437a301288969109c2) and identified K-type by using Kleborate (https:/ / github.com/katholt/Kleborate). We further detected mutations in rmpA and rmpA2 by using blastn (https://blast.ncbi.nlm.nih.gov/Blast.cgi). We included genome assemblies of the isolates sequenced in this study and the 62 isolates published elsewhere (10-12,24-26) in the phylogenetic and temporal analysis (Appendix 2 Table 1, https://wwwnc.cdc.gov/ EID/article/26/2/19-0594-App2.xlsx).

\section{Statistical Analysis}

Statistical analyses are described in Appendix 1. We conducted all statistical analyses by using SPSS Statistics 23 (IBM, https://www.ibm.com) and SAS 9.4 (SAS institute, https://www.sas.com).

\section{Results}

\section{Capsular Switch in CRKP-ST11 over a 4-Year Period}

We retrospectively screened 10,134 K. pneumoniae isolates to determine the proportion of BSI-CRKP. Of 705 nonrepetitive bloodstream isolates, 203 were CRKP. The proportion of K. pneumoniae and CRKP in BSIs increased from $17.1 \%$ to $45.5 \%$ during the study period (Table 1). ST11 was the predominant 
Table 1. Prevalence trend of Klebsiella pneumoniae causing BSIs in a tertiary hospital, China, 2013-2017*

\begin{tabular}{|c|c|c|c|c|c|c|c|}
\hline Isolate type & 2013 & 2014 & 2015 & 2016 & 2017 (half year) & $\begin{array}{l}\text { Score test } \\
\text { for trend }\end{array}$ & $\mathrm{p}$ value \\
\hline Primary $\mathrm{BSI} /$ non-BSI isolates & $123 / 610$ & $133 / 635$ & $201 / 723$ & $149 / 687$ & $99 / 398$ & 1.3934 & 0.1635 \\
\hline CRKP/non-CRKP & $21 / 102$ & $35 / 98$ & $53 / 148$ & $49 / 100$ & $45 / 54$ & 6.0697 & $<0.001$ \\
\hline CRKP-ST11/non-ST11 CRKP & $20 / 1$ & $28 / 7$ & $46 / 7$ & $39 / 10$ & $41 / 4$ & -0.3116 & 0.7553 \\
\hline ST11-KL47/ST11-KL64 & $18 / 1$ & $20 / 4$ & $22 / 23$ & $11 / 28$ & $5 / 36$ & -7.5463 & $<0.001$ \\
\hline
\end{tabular}

clone among BSI-CRKP isolates, accounting for $85.7 \%$ ( $\mathrm{n}=174)$; annual distribution was relatively stable $(95.2 \%-91.1 \%)$.

Five KLs were detected in the BSI-CRKP-ST11 population: KL47 ( $\mathrm{n}=76), \operatorname{KL} 64(\mathrm{n}=92)$, KL31 (n $=3), \operatorname{KL103}(\mathrm{n}=2)$, and KL105 $(\mathrm{n}=1)$. The ratio of ST11-KL47 to CRKP-ST11 dropped from 90\% (18/20) in 2013 to $12.2 \%$ (5/41) in 2017, whereas that of ST11KL64 increased from $4.6 \%(1 / 20)$ in 2013 to $87.8 \%$ (36/41) in 2017. Thus, the ratio of ST11-KL47 to ST11KL64 decreased substantially in the study period (Table 1), suggesting a KL shift among the CRKP-ST11 population over the 4 -year period.

\section{ST11-KL64 Infections as Cause of Higher 30-Day Mortality}

To evaluate the clinical importance of ST11-KL47 and ST11-KL64, we analyzed 162 ST11-infected patients with complete clinical data, 72 patients with ST11-KL47 and 90 with ST11-KL64 (Appendix 2 Table 2); 4 ST11-KL47-infected and 2 ST11-KL64infected outpatients were excluded. ST11-KL47 patients had a significantly longer stay than did ST11KL64 patients, with respect to both the total hospital stay $(\mathrm{p}=0.001)$ and hospital stay before the BSI onset $(p=0.029)$. More ST11-KL47-infected patients acquired lung infections and received invasive procedures, devices, or both before and after BSI; they also had received hemodialysis and chemotherapy or radiotherapy within 30 days before BSI. However, the Charlson comorbidity score was identical for patients of both groups. Patients infected with ST11-KL64 showed significantly higher overall 30day mortality than those with ST11-KL47 (62.2\% vs. $52.8 \% ; 2=4.252 ; \mathrm{p}=0.039$ ) (Figure 1 ).

We further included 29 patients infected with nonST11 CRKP in the analysis to evaluate whether CRKPST11 caused higher mortality than non-ST11 CRKP. We found no significant differences in 30-day mortality between patients infected with CRKP-ST11 and those with non-ST11 CRKP $\left(57.1 \%\right.$ vs. $44.8 \% ; \chi^{2}=0.833 ; p=$ 0.176). Cox regression multivariate analysis revealed 3 factors independently associated with a higher risk for ST11-caused mortality: lower platelet at time of BSI, Acute Physiology and Chronic Health Evaluation
(APACHE II) score, and tigecycline as the empirical therapy (Table 2; Appendix 2 Table 3). We also found no significant difference in 30-day mortality between patients infected with ST11-KL47 and those with nonST11 CRKP (52.8\% vs. $44.8 \% ; 2=0.395 ; \mathrm{p}=0.529$ ). However, the ST11-KL64-infected patients showed significantly higher 30-day mortality than those with non-ST11 CRKP ( $62.2 \%$ vs. $44.8 \% ; 2=3.771 ; \mathrm{p}=0.05)$.

\section{Recombination-Mediated Evolutionary Diversification in CRKP-ST11}

We performed phylogenomic analysis to understand the evolutionary diversification in the CRKP-ST11 population. We included 154 newly sequenced genomes (excluding the remaining 20 isolates without rmpA or rmpA2); 62 previously published ST11 genomes from diverse origins; and an ST1731 genome (accession no. ERR1541319) as an outgroup. We identified 429 recombined regions, including 348 that were $>1 \mathrm{~kb}$. The length of sequence removed per isolate ranged from 505,312 to 1,276,214 bp (median 947,836 $\mathrm{bp}$ ). The phylogenetic tree, which was rooted using the ST1731 outgroup isolate that was later removed (Appendix 1 Figure 1), showed division of ST11 isolates into 2 major clades (Figure 2). One clade consists of isolates of KL47, KL64, and KL31 exclusively obtained from China, whereas the second clade consists of isolates possessing diverse K-types from elsewhere. These findings suggest that KL47 and KL64 have emerged and undergone local expansion in China.

Root-to-tip regression analysis of the 154 newly sequenced genomes demonstrated a correlation between the genetic distances and sampling dates $\left(\mathrm{R}^{2}=0.64\right)$ (Appendix 1 Figure 2). By using a Bayesian dating method implemented in BactDating (27), we found that KL64 isolates probably evolved from a KL47 ancestor around 2011 (Appendix 1 Figure 3). A high substitution rate also was found (15.3 singlenucleotide polymorphisms (SNPs)/genome/y, 95\% CI 12.4-19.0 SNPs/genome/y).

The number of SNPs separating ST11-KL47 and ST11-KL64 isolates was 907-3,098 before and 30-220 after removal of recombination regions. This finding suggests that recombination largely contributed to the diversification of ST11-KL47 and ST11-KL64. Indeed, 


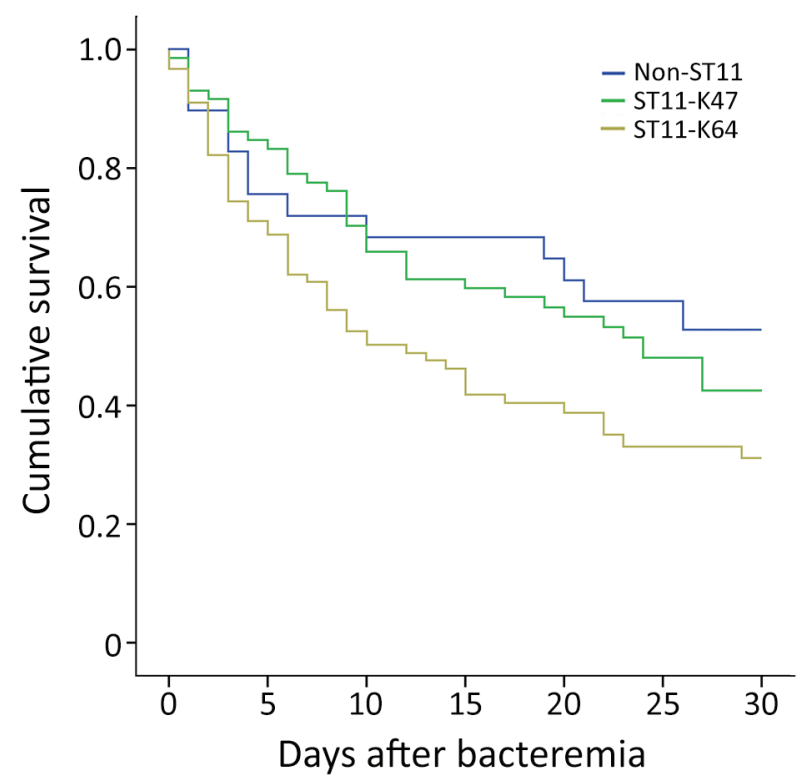

Figure 1. Kaplan-Meier survival estimates for patients with bloodstream infections caused by ST11-KL47, ST11-KL64, and non-ST11 CRKP, China, 2013-2017. A significant difference was found in the 30-day mortality among the 3 groups $(p=0.039$ ). ST11-KL64-infected patients showed significantly higher overall 30 -day mortality than ST11-KL47-infected patients $(62.2 \%$ vs. $52.8 \% ; p=0.039$ ) and non-ST11 CRKP-infected patients $(62.2 \%$ vs. $44.8 \%$; $p=0.05)$. No significant difference in 30 -day mortality was found between patients infected with ST11-KL47 and non-ST11 CRKP (52.8\% vs. $44.8 \%, p=0.529)$. CRKP, carbapenem-resistant Klebsiella pneumoniae; KL, capsular loci; ST, sequence type.

we detected 4 recombination events of $>1 \mathrm{~kb}$ on the branch coinciding with the switch from KL47 to KL64 (regions with respect to the reference genome [isolate KP47434]: 307,448-322,057; 4,060,806-4,154,013; 4,173,036-4,186,742; and 4,197,111-4,217,597) (Appendix 1 Figure 4; Appendix 2 Table 4). Three of these events were localized around the cps region, suggesting that the capsule switch was likely the result of recombination. Another recombination event in the cps region also corresponds with the capsule switch from KL47 to KL31 (Appendix 1 Figure 4).

\section{Emergence of $r m p A-r m p A 2-P o s i t i v e$ ST11-KL64 Isolates}

Analysis of virulence genes showed that the 154 ST11 isolates possessed yersiniabactin genes (ybtAEPQSTUX, irp1, irp2, and fyuA) located on ICEKp3, and the core type III fimbrial cluster mrkABCDF, except for 4 ST11-KL64 isolates. Of 76 ST11-KL47 isolates, 29 $(36.3 \%)$ carried an rmpA2 gene, and only 2 were positive for the string test, suggesting that rmpA2 was inactive in most isolates. rmpA2-positive ST11-KL47 isolates have been detected since 2013 and are interspersed
Table 2. Multivariable analysis of risk factors for 30-day mortality in $191 \mathrm{BSI}$ patients infected with carbapenem-resistant Klebsiella pneumoniae, China, 2013-2017*

\begin{tabular}{lcc}
\hline Variable & p value† & OR $(95 \% \mathrm{Cl})$ \\
\hline Platelets at time of BSI & 0.001 & $\begin{array}{c}0.996 \\
(0.994-0.998)\end{array}$ \\
\hline APACHE II score at time of BSI & 0.012 & $\begin{array}{c}1.041 \\
(1.009-1.074\end{array}$ \\
\hline Tigecycline as empirical therapy & 0.003 & 1.920 \\
& & $(1.257-2.935)$ \\
\hline $\begin{array}{l}\text { *APACHE, Acute Physiology and Chronic Health Evaluation; BSI, } \\
\text { bloodstream infection; OR, odds ratio. } \\
\text { tCalculated by using Cox regression. }\end{array}$ & \\
\hline
\end{tabular}

among rmpA2-negative isolates in the phylogenetic tree (Figure 2). A frameshift rmpA2 gene (rmpA2*) was identified in 48 of 92 ST11-KL64 isolates (52.2\%). rmpA2*-positive ST11-KL64 isolates were first detected in 2015, and most of them are monophyletic (Figure 2). An rmpA gene was also found in 42 of the 48 rmpA2*positive ST11-KL64 isolates, of which 6 had in-frame truncations resulting in 2 variants (555 bp and 624 $\mathrm{bp)}$ and 12 were positive for the string test. The rmpArmpA2*-positive ST11-KL64 isolates were detected from 2016 onward. The prevalence trend of rmpA/ rmpA2-positive isolates is accordant with that of each subclone (Appendix 1 Figure 5).

All $r m p A / r m p A 2 *$-positive isolates also carried aerobactin genes iucABCD-iutA, implying that they might co-locate on the same plasmid. A plasmidborne virulence factor peg-344 was exclusively found in 45 of $48 \mathrm{rmpA2}$-positive ST11-KL64 isolates. The salmochelin cluster iroBCDN was also detected in 5 rmpA-rmpA2*-positive and in 1 classic ST11-KL64 isolate (Figure 2).

\section{Diversity of Virulence Plasmids}

We further analyzed the vectors of $r m p A / r m p A 2$ genes to understand how they were captured. The rmpA/rmpA2 gene of both subclones was detected on plasmids by using southern blot (Appendix 1 Figure 6). Higher diversity of the virulence plasmids was found in ST11-KL64 through classifying the plasmids by size; 5 types were detected in ST11-KL47 with sizes ranging from 110 to $217 \mathrm{~kb}$, and 13 types were in ST11-KL64, ranging from 110 to $230 \mathrm{~kb}$ (Appendix 2 Table 5). The rmpA and rmpA2* genes coexisted on the same plasmid in ST11-KL64.

Virulence plasmids detected in KP16932 (KL47) and KP47434 (KL64) were circularized to evaluate their structural variations. The rmpA2 gene of KP16932 was carried by an IncFIB(K)-IncHI1B-type plasmid (pVir-KP16932) with a size of $177.8 \mathrm{~kb}$, which is almost identical to a virulence plasmid pVir-CR-HvKP4 (MF437313) recently detected in a KL47 clone that caused a fatal outbreak in China (Appendix 1 Figure 

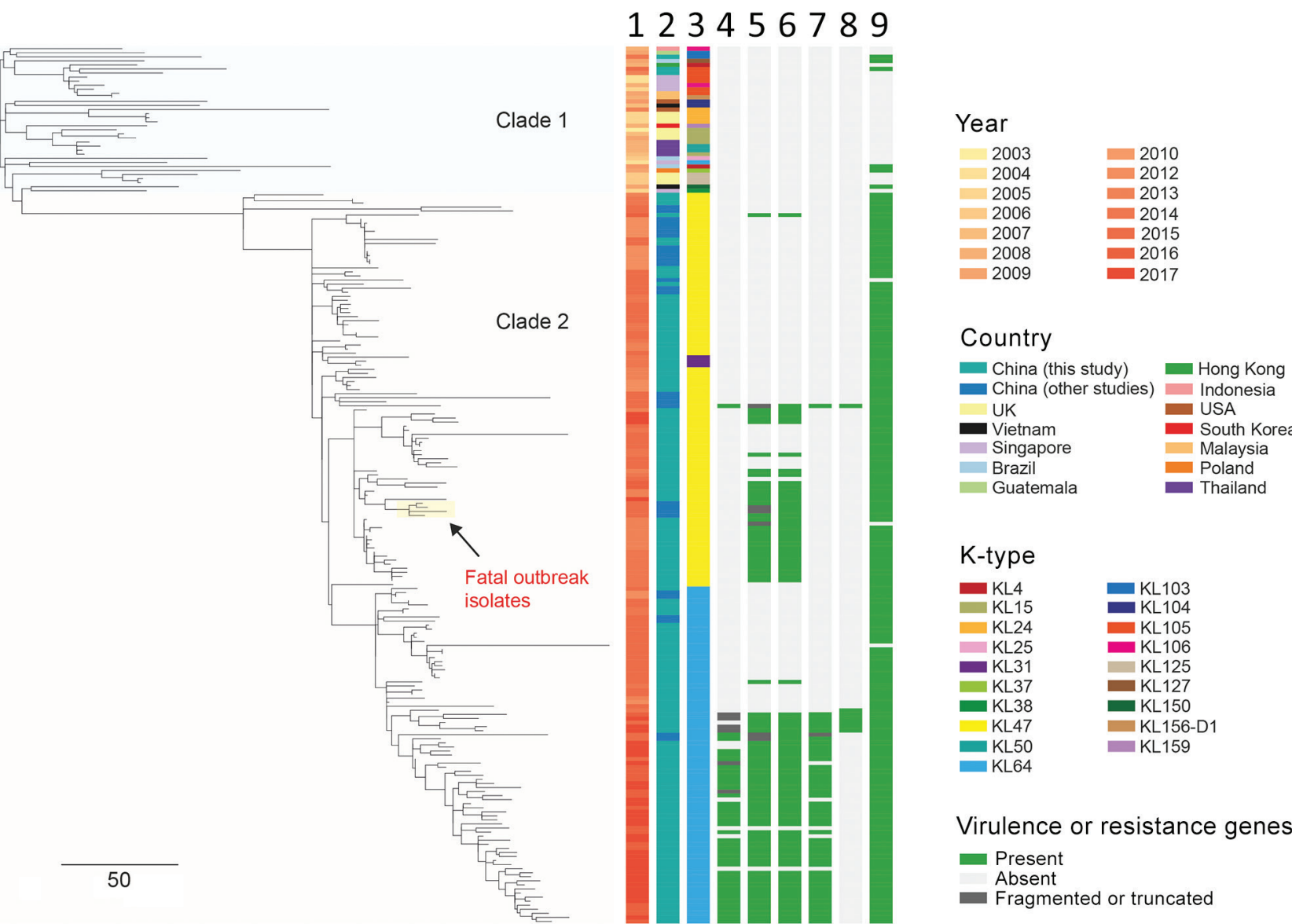

Figure 2. Phylogenetic analysis of 216 CRKP ST11 isolates, China, 2013-2017, including 154 CRKP isolates collected during 2012-2017 in study of bloodstream infections in a tertiary hospital and 62 isolates that were sequenced in previous studies (Appendix 2 Table 1 , https://wwwnc.cdc.gov/EID/article/26/2/19-0594-App2.xlsx). The phylogenetic tree was obtained by mapping all sequence reads to the hybrid assembly of KP47434 and removing the recombined regions from the alignment. The tree was rooted using ST1731 isolate EuSCAPE_ES29 (ERR1541319), which was included in this analysis but later removed from the tree (a tree including this outgroup is shown in Appendix 1 Figure 1, https://wwwnc.cdc.gov/EID/article/26/2/19-0594-App1.pdf). Five capsular types (KL31, KL47, KL64, KL103, and KL105) were detected in our ST11 collection, which are indicated in different colors as shown in the legend. Some of virulence genes detected are shown here. The $r m p A 2$ gene carried by KL64 isolates was frameshifted, namely rmpA2*. Aerobactin and salmochelin represent the iucABCD-iutA and iroBCDN gene clusters, respectively. The fatal outbreak clone reported in China recently (12) is highlighted on the tree. Lanes: 1 , year; 2, country; 3, K-type; 4, rmpA; 5, rmpA2; 6, aerobactin; 7, peg-344; 8, salmochelin; 9, blaKPC. Scale bar indicates single-nucleotide polymorphisms. CRKP, carbapenem-resistant Klebsiella pneumoniae; KL, capsular loci; ST, sequence type.

7). The rmpA and rmpA2* genes of KP47434 existed in an IncFIB(K)-IncHI1B-type plasmid (pVir-KP47434) with a size of $201.8 \mathrm{~kb}$, which shares a high homology with a virulence plasmid pVir-CR-HvKP267 (accession no. MG053312). Compared with pVir-KP47434, a 24$\mathrm{kb}$ and an 18-kb region were absent in pVir-KP16932 (Appendix 1 Figure 7), which encodes genes involved in metabolic processes such as carbon utilization (OppA-B-F and DppC) (28) and virulence (H-NS protein) (29). The virulence plasmids carried by rmpA2-KL47 and $r m p A / r p m A 2 *$ KL64 isolates possessed the highly similar backbone sequences with the pVir-KP16932 and pVir-KP47434 plasmids, respectively, and the intra-subclonal variations were mainly caused by gain or loss of gene clusters involved in heavy metal resistance and mobile genetic elements (Appendix 1 Figure 8).

\section{Virulence Plasmids and Infections}

To evaluate whether the acquisition of virulence plasmids carrying $r m p A / r m p A 2$ and aerobactin genes has an effect on clinical outcomes, we stratified the cohort described according to the existence of virulence plasmids in ST11-KL47 and ST11-KL64. No significant differences in mortality were evident between patients infected by ST11-KL64-pVir-KP47434-like or classical ST11-KL64 isolates (i.e., without virulence plasmids) 
(60.4\% vs. $64.3 \%$; $\mathrm{p}=0.983)$ or those infected by ST11KL47-pVir-KP16932 or classical ST11-KL47 isolates (51.7\% vs. 53.5\%; $\mathrm{p}=0.931$ ) (Appendix 2 Table 6, 7). However, the Charlson comorbidity scores for patients with ST11-KL47-pVir-KP16932-like infections (median 1 [range 0.5-2]) were significantly lower than scores for patients with ST11-KL47 infections (median 3 [range 1-4]; $p=0.003$ ); similarly, scores for ST11KL64-pVir-KP47434-like patients (median 1 [range $0-2])$ were significantly lower than scores for ST11KL64 patients (median 2 [range 1-3]; $p=0.003$ ). These findings indicate that the virulence plasmids could promote infections in healthier patients.

\section{Various Resistomes in ST11-KL47 and ST11-KL64}

The resistome of ST11-KL47 was different from that of ST11-KL64 (Appendix 1 Figure 9). Genes floR, arr-3, dfrA27, and aac(6')-Ib-cr were exclusively detected in ST11-KL47, whereas blaSHV-12 and dfrA14 were unique for ST11-KL64, suggesting the 2 subclones might have been selected for in different niches. For each subclone, the resistomes of rmpA/ rmpA2-positive isolates were much more consistent than those of classical isolates (Appendix 1 Figure 9). This finding is consistent with the phylogeny and the fact that the $r m p A / r m p A 2$-positive isolates were relatively more clonal.

\section{Enhanced Virulence in ST11-KL64}

The $r m p A / r m p A 2$-encoding virulence plasmids carried by each subclone shared an intrasubclonal similarity as described previously (i.e., the intrasubclonal variations were mainly caused by gain or loss of heavy metal resistance gene clusters and mobile genetic elements). ST11-KL64 isolates produced significantly more biofilm than ST11-KL47 isolates (optical density at $595 \mathrm{~nm}$ : $0.54+$ SD 0.09 vs. $3.08+$ SD 0.11; $<<0.0001$ ) (Appendix 1 Figure 10, panel A). We evaluated the virulence potential by using a human neutrophil assay. The ST11-KL64 isolates had an average survival of $91.2 \%$ after incubation with the human neutrophils for $60 \mathrm{~min}$, which was significantly higher than that of the ST11-KL47 strains $(65.8 \% ; p=0.0011)$ (Appendix 1 Figure 10, panel B). Compared with the ST11-KL64 isolates, the ST23-K1 isolates showed lower survival (70.3\%) and the ST86K2 isolates comparable survival (91.8\%). The ST35 isolate had the lowest survival, $37.2 \%$.

We further estimated pathogenicity by infecting $G$. mellonella larvae with an inoculum of $1 \times 106$ CFU. At $48 \mathrm{~h}$ postinfection, the 4 ST11-KL64 isolates (1 classic [isolate KP33068], $1 \mathrm{rmpA2*}$-positive [isolate KP33130], and 2 rmpA-rmpA2*-positive [isolates KP33229 and KP33367]) showed comparable virulence resulting in
$10 \%$ survival, whereas survival was $40 \%-60 \%$ for the 4 ST11-KL47 isolates (2 classic [isolates KP9343 and KP29407] and 2 rmpA2-positive [isolates KP10042 and KP16932]). K1 survival 40\% and K2 30\%; survival of a classic CRKP isolate (ST35) reached 70\% (Appendix 1 Figure 10, panel C).

To determine the underlying mechanisms of enhanced transmissibility obtained by $\operatorname{rmpA-rmpA2*}$ positive ST11-KL64, we randomly selected 6ST11-KL47 isolates (3 classic [KP8369, KP29407, and KP30412] and 3 rmpA2-positive [KP9343, KP10042, and KP16932]) and 6 ST11-KL64 isolates (2 classic [KP28367 and KP33068], 2 rmpA2*-positive [KP33130 and KP45812], and 2 rmpArmpA2*-positive [KP47434 and KP39615]) to evaluate the capacity of survival on a dry polystyrene surface. Only viable cells of $2 r m p A-r m p A 2^{*}$-KL64 isolates were recovered after overnight drying; the average recovered loads were $90+31.09 \mathrm{CFU} / \mathrm{mL}$ and $115+20.62 \mathrm{CFU} /$ $\mathrm{mL}$. This finding suggests that the enhanced transmissibility of the newly emerged subclone was associated with enhanced environmental survival.

\section{National Prevalence of BSI-CRKP-ST11}

To estimate the national prevalence of BSI-CRKPST11, we further retrospectively screened 1,098 clinical BSI-KP strains collected from 13 provinces in China during 2014-2016 (Table 3). In total, 46 of 83 CRKP strains were ST11; ST11-KL47 accounted for $80.4 \%$ and ST11-KL64 19.6\%. The rmpA2 gene was detected in 11 ST11-KL47 and 1 ST11-KL64 isolates, and 1 isolate of each subclone also co-harbored an rmpA gene. The $r m p A 2$-positive isolates were detected from Anhui and Zhejiang provinces. Most (9/12) rmpA2positive CRKP isolates appeared after 2015.

\section{Discussion}

The global dissemination of CRKP poses a serious threat to public health. Control of CRKP in populations and healthcare networks thus becomes an urgent issue. However, efforts are often complicated by rapid evolution, especially among epidemic clones (e.g., ST11 and ST258). Therefore, tracking of evolutionary events and understanding their clinical importance are critical. We performed a comprehensive study to provide insight into the evolution of key virulence features of BSI-CRKP collected in China. We found 2 major KLs (KL47 and KL64) in the dominant clone BSI-CRKP-ST11. Capsule is known as an important immune-evasion molecule, and thus has become a popular target for vaccine design. Determining the prevalence of KLs is crucial for the development of capsule-based vaccines and phage-derived exopolysaccharide-depolymerase treatments, which are 
Table 3. Prevalence of CRKP-ST11 causing BSIs, China, 2014-2016*

\begin{tabular}{lccccc}
\hline Year & KP & CRKP & CRKP-ST11 & $\begin{array}{c}\text { CRKP-ST11-KL47, } \\
\text { rmpA/rmpA2-positive }\end{array}$ & $\begin{array}{c}\text { CRKP-ST11-KL64, } \\
\text { rmpA/rmpA2-positive }\end{array}$ \\
\hline 2014 & 224 & 10 & 7 & $5(1)$ & $2(0)$ \\
2015 & 345 & 31 & 19 & $16(2)$ & $3(0)$ \\
2016 & 529 & 42 & 20 & $16(8)$ & $4(1)$ \\
\hline Total & 1,098 & 83 & 46 & $37(11)$ & $9(1)$ \\
\hline *Isolates were collected as part of national surveillance for BSIs. BSI, bloodstream infection; CRKP, carbapenem-resistant Klebsiella pneumoniae; \\
KL, capsular loci; KP, Klebsiella pneumoniae; ST, sequence type.
\end{tabular}

considered as novel approaches for the treatment of CRKP infections (30). Our study provides useful data for assisting the development of an immunotherapy for ST11-CRKP infections in China.

In this study, ST11 was partitioned into 2 clades, 1 consisting of ST11-KL47, ST11-KL64, and ST11KL31, suggesting that these strains were diversified from a common ancestor. We found that sequences within the cps region of ST11-KL64 and ST11-KL31 were imported through recombination indicating the occurrence of capsule switching. By using a Bayesian approach, we found that ST11-KL64 might have emerged from an ST11-KL47-like ancestor in 2011. We further noted that the 2 ST11 subclones (ST11-KL47 and ST11-KL64) have spread nationally by interregional transmission. However, the lack of genome data about ST11-KL47 and ST11-KL64 from different origins hampers our understanding of spatial evolution at a global scale.

The notion of a rapid evolution of the ST11 population is supported by numerous cps variants ( $\mathrm{n}=$ $19)$ and the very high evolutionary rate (15.3 SNPs/ genome/year) detected in this study and others (9). Capsule switching has been suggested to be a common event across the wider K. pneumoniae population through large recombination events (9-11). We suppose that generating numerous descendants with various combinations of evolved chromosomes and capsules heavily contributes to the success of ST11 and its descendants (e.g., ST258). Of note, our study identified a clonal replacement in the CRKPST11 population over a 4-year period in a hospital. ST11-KL47, the dominant subclone before 2015, was progressively replaced by ST11-KL64. The population structure of ST11-K64 was monophyletic, implying that ST11-KL64 might have gained fitness and was ready to disseminate clonally like ST258. Also, KL64 is a more commonly observed capsule type than KL47, and has been detected in Brazil (25), Taiwan (31), Singapore (32), the United States, and Europe (33).

To understand the clinical importance of the clonal replacement that coincided with the capsular switch in the ST11 population, we analyzed the metadata of 162 infected patients. Patients infected by ST11-KL64 had significantly higher mortality rates than those infected by ST11-KL47 and non-ST11 CRKP. This finding is supported by the results of our phenotypic assays, which showed that ST11-KL64 was more virulent than ST11-KL47. Our findings suggest that the acquisition of virulence plasmids promotes the infection in healthier patients but is not associated with the increased mortality, indicating that other virulence factors might be involved. Capsular switching in the ST11 population might contribute to increased mortality. The capsule type is thought to be an important determinant for the pathogenicity of K. pneumoniae, like the notorious capsular serotypes $\mathrm{K} 1$ and K2. Similar associations are also identified in other species, such as Acinetobacter baumannii (34) and Streptococcus spp. $(35,36)$. We also cannot exclude that the enhanced virulence and increased mortality might be associated with other chromosomal and plasmid variations.

We further noted that the newly emerged $r m p A 2 *$ positive ST11-KL64 isolates exclusively carried an rmpA gene. The presence of a truncated variant might confer an advantage through a more subtle activation of capsule expression in comparison to a strain with 2 fully functional variants present (37). In addition, the combination of RmpA and truncated $R m p A 2$ was previously found predominantly in clinical isolates with a hypervirulent or hypermucoviscous phenotype (38). This finding is consistent with our study, given that rmpA-rmpA2*-ST11-KL64 isolates become the dominant clone after they emerged. We suppose that such combination might confer fitness to the population resulting in the replacement of rmpA2ST11-KL47 by rmpA-rmpA2*-ST11-KL64. This supposition can be supported by the fact that rmpA-rmpA2*ST11-KL64 isolates survive longer than ST11-KL47 in vitro, which largely facilitates a better dissemination of the population under nosocomial conditions. Besides the isolates found in Anhui and Zhejiang provinces in our study, 2 rmpA-rmpA2*-ST11-KL64 isolates have been detected in Shanghai and Henan provinces (9), suggesting that the newly emerged subclone has widely disseminated in China.

In summary, our study identified the emergence of a high-risk subclone of CRKP-ST11, resulting in 
enhanced virulence and transmissibility. The newly emerging descendant obtained enhanced environmental survival and poses a substantial threat to healthcare networks, suggesting the urgent need for tailor-made surveillance and stricter infection-control measures to prevent further dissemination in nosocomial settings.

\section{Acknowledgments}

We thank Jinru Ji and Chaoqun Ying for their assistance during sample collection and data analysis.

This work was supported by the National Key Research and Development Program of China (grant no.

2017YFC1200200), Major Infectious Diseases such as AIDS and Viral Hepatitis Prevention and Control Technology Major Projects (grant no. 2018ZX10712-001), and the National Natural Science Foundation of China (81702045, 81361138021).

We deposited the 2 scaffolded genome sequences in GenBank under the accession nos. QVAN00000000 (KP16932) and QURI00000000 (KP47434); accession numbers of the other genome sequences are listed in Appendix 2 Table 1 . The datasets supporting the conclusions of this article are included in the article and in Appendix 2.

\section{About the Author}

Dr. Kai Zhou is an associate professor at the First Affiliated Hospital of Southern University of Science and Technology, Shenzhen, China. His research interests are epidemiology and drug-resistance mechanisms of carbapenem-resistant Enterobacteriaceae.

\section{References}

1. Guh AY, Bulens SN, Mu Y, Jacob JT, Reno J, Scott J, et al. Epidemiology of carbapenem-resistant Enterobacteriaceae in 7 US communities, 2012-2013. JAMA. 2015;314:1479-87. https://doi.org/10.1001/jama.2015.12480

2. Grundmann H, Glasner C, Albiger B, Aanensen DM, Tomlinson CT, Andrasević AT, et al.; European Survey of Carbapenemase-Producing Enterobacteriaceae (EuSCAPE) Working Group. Occurrence of carbapenemase-producing Klebsiella pneumoniae and Escherichia coli in the European survey of carbapenemase-producing Enterobacteriaceae (EuSCAPE): a prospective, multinational study. Lancet Infect Dis. 2017;17:153-63. https://doi.org/10.1016/ S1473-3099(16)30257-2

3. Zhang R, Liu L, Zhou H, Chan EW, Li J, Fang Y, et al. Nationwide surveillance of clinical carbapenem-resistant Enterobacteriaceae (CRE) strains in China. EBioMedicine. 2017;19:98-106. https:// doi.org/10.1016/j.ebiom.2017.04.032

4. Patel G, Huprikar S, Factor SH, Jenkins SG, Calfee DP. Outcomes of carbapenem-resistant Klebsiella pneumoniae infection and the impact of antimicrobial and adjunctive therapies. Infect Control Hosp Epidemiol. 2008;29:1099-106. https:// doi.org/10.1086/592412
5. Pitout JDD, Nordmann P, Poirel L. Carbapenemaseproducing Klebsiella pneumoniae, a key pathogen set for global nosocomial dominance. Antimicrob Agents Chemother. 2015;59:5873-84. https://doi.org/10.1128/AAC.01019-15

6. Chen L, Mathema B, Pitout JDD, DeLeo FR, Kreiswirth BN. Epidemic Klebsiella pneumoniae ST258 is a hybrid strain. MBio. 2014;5:e01355-14.

7. Gaiarsa S, Comandatore F, Gaibani P, Corbella M, Dalla Valle C, Epis S, et al. Genomic epidemiology of Klebsiella pneumoniae in Italy and novel insights into the origin and global evolution of its resistance to carbapenem antibiotics. Antimicrob Agents Chemother. 2015;59:389-96.

8. DeLeo FR, Chen L, Porcella SF, Martens CA, Kobayashi SD, Porter AR, et al. Molecular dissection of the evolution of carbapenem-resistant multilocus sequence type 258 Klebsiella pneumoniae. Proc Natl Acad Sci U S A. 2014;111:4988-93. https://doi.org/10.1073/pnas.1321364111

9. Wyres KL, Gorrie C, Edwards DJ, Wertheim HFL, Hsu LY, Van Kinh N, et al. Extensive capsule locus variation and large-scale genomic recombination within the Klebsiella pneumoniae clonal group 258. Genome Biol Evol. 2015;7: 1267-79. https://doi.org/10.1093/gbe/evv062

10. Jiang Y, Wei Z, Wang Y, Hua X, Feng Y, Yu Y. Tracking a hospital outbreak of KPC-producing ST11 Klebsiella pneumoniae with whole genome sequencing. Clin Microbiol Infect. 2015;21:1001-7. https:// doi.org/10.1016/j.cmi.2015.07.001

11. Dong N, Zhang R, Liu L, Li R, Lin D, Chan EW-C, et al. Genome analysis of clinical multilocus sequence type 11 Klebsiella pneumoniae from China. Microb Genom. 2018;4:5412. https:// doi.org/10.1099/mgen.0.000149

12. Gu D, Dong N, Zheng Z, Lin D, Huang M, Wang L, et al. A fatal outbreak of ST11 carbapenem-resistant hypervirulent Klebsiella pneumoniae in a Chinese hospital: a molecular epidemiological study. Lancet Infect Dis. 2018;18:37-46. https://doi.org/10.1016/S1473-3099(17)30489-9

13. Du P, Zhang Y, Chen C. Emergence of carbapenemresistant hypervirulent Klebsiella pneumoniae. Lancet Infect Dis. 2018;18:23-4. https:// doi.org/10.1016/ S1473-3099(17)30625-4

14. Yao H, Qin S, Chen S, Shen J, Du X-D. Emergence of carbapenem-resistant hypervirulent Klebsiella pneumoniae. Lancet Infect Dis. 2018;18:25. https://doi.org/10.1016/ S1473-3099(17)30628-X

15. Wong MHY, Shum H-P, Chen JHK, Man M-Y, Wu A, Chan EW-C, et al. Emergence of carbapenem-resistant hypervirulent Klebsiella pneumoniae. Lancet Infect Dis. 2018;18:24. https:/ / doi.org/10.1016/S1473-3099(17)30629-1

16. Xiao T, Yu W, Niu T, Huang C, Xiao Y. A retrospective, comparative analysis of risk factors and outcomes in carbapenem-susceptible and carbapenem-nonsusceptible Klebsiella pneumoniae bloodstream infections: tigecycline significantly increases the mortality. Infect Drug Resist. 2018;11:595-606. https:/ / doi.org/10.2147/IDR.S153246

17. Diancourt L, Passet V, Verhoef J, Grimont PAD, Brisse S. Multilocus sequence typing of Klebsiella pneumoniae nosocomial isolates. J Clin Microbiol. 2005;43:4178-82. https://doi.org/10.1128/JCM.43.8.4178-4182.2005

18. McLaughlin MM, Advincula MR, Malczynski M, Barajas G, Qi C, Scheetz MH. Quantifying the clinical virulence of Klebsiella pneumoniae producing carbapenemase Klebsiella pneumoniae with a Galleria mellonella model and a pilot study to translate to patient outcomes. BMC Infect Dis. 2014;14:31. https:/ / doi.org/10.1186/1471-2334-14-31

19. Naparstek L, Carmeli Y, Navon-Venezia S, Banin E. Biofilm formation and susceptibility to gentamicin and colistin of extremely drug-resistant KPC-producing Klebsiella 
pneumoniae. J Antimicrob Chemother. 2014;69:1027-34. https://doi.org/10.1093/jac/dkt487

20. Wang L, Shen D, Wu H, Ma Y. Resistance of hypervirulent Klebsiella pneumoniae to both intracellular and extracellular killing of neutrophils. PLoS One. 2017;12:e0173638. https:// doi.org/10.1371/journal.pone.0173638

21. Havill NL, Boyce JM, Otter JA. Extended survival of carbapenem-resistant Enterobacteriaceae on dry surfaces. Infect Control Hosp Epidemiol. 2014;35:445-7. https:// doi.org/10.1086/675606

22. Wick RR, Judd LM, Gorrie CL, Holt KE. Unicycler: Resolving bacterial genome assemblies from short and long sequencing reads. PLoS Comput Biol. 2017;13:e1005595. https://doi.org/10.1371/journal.pcbi.1005595

23. Henson SP, Boinett CJ, Ellington MJ, Kagia N, Mwarumba S, Nyongesa S, et al. Molecular epidemiology of Klebsiella pneumoniae invasive infections over a decade at Kilifi County Hospital in Kenya. Int J Med Microbiol. 2017;307:422-9. https:/ / doi.org/10.1016/j.ijmm.2017.07.006

24. Holt KE, Wertheim H, Zadoks RN, Baker S, Whitehouse CA, Dance D, et al. Genomic analysis of diversity, population structure, virulence, and antimicrobial resistance in Klebsiella pneumoniae, an urgent threat to public health. Proc Natl Acad Sci U S A. 2015;112:E3574-81. https:/ / doi.org/10.1073/ pnas.1501049112

25. Bowers JR, Kitchel B, Driebe EM, MacCannell DR, Roe C, Lemmer D, et al. Genomic analysis of the emergence and rapid global dissemination of the clonal group 258 Klebsiella pneumoniae pandemic. PLoS One. 2015;10:e0133727. https://doi.org/10.1371/journal.pone.0133727

26. Moradigaravand D, Martin V, Peacock SJ, Parkhill J. Evolution and epidemiology of multidrug-resistant Klebsiella pneumoniae in the United Kingdom and Ireland. MBiol 2017;8:e01976-16.

27. Didelot X, Croucher NJ, Bentley SD, Harris SR, Wilson DJ. Bayesian inference of ancestral dates on bacterial phylogenetic trees. Nucleic Acids Res. 2018;46:e134-4. https://doi.org/10.1093/nar/gky783

28. Lee E-M, Ahn S-H, Park J-H, Lee J-H, Ahn S-C, Kong I-S. Identification of oligopeptide permease (opp) gene cluster in Vibrio fluvialis and characterization of biofilm production by oppA knockout mutation. FEMS Microbiol Lett. 2004;240:2130. https:// doi.org/10.1016/j.femsle.2004.09.007

29. Ares MA, Fernández-Vázquez JL, Rosales-Reyes R, Jarillo-Quijada MD, von Bargen K, Torres J, et al. H-NS nucleoid protein controls virulence features of Klebsiella pneumoniae by regulating the expression of type 3 pili and the capsule polysaccharide. Front Cell Infect Microbiol. 2016;6:13. https://doi.org/10.3389/fcimb.2016.00013

30. Kobayashi SD, Porter AR, Freedman B, Pandey R, Chen L, Kreiswirth BN, et al. Antibody-mediated killing of carbapenemresistant ST258 Klebsiella pneumoniae by human neutrophils. MBio. 2018;9:1198. https:/ / doi.org/10.1128/mBio.00297-18

31. Pan Y-J, Lin T-L, Lin Y-T, Su P-A, Chen C-T, Hsieh P-F, et al. Identification of capsular types in carbapenemresistant Klebsiella pneumoniae strains by wzc sequencing and implications for capsule depolymerase treatment. Antimicrob Agents Chemother. 2015;59:1038-47. https:/ / doi.org/10.1128/AAC.03560-14

32. Koh TH, Cao D, Shan QY, Bacon A, Hsu LY, Ooi EE. Acquired carbapenemases in Enterobactericeae in Singapore, 1996-2012. Pathology. 2013;45:600-3. https:/ / doi.org/ 10.1097/PAT.0b013e3283650b1e

33. Cryz SJ Jr, Mortimer PM, Mansfield V, Germanier R. Seroepidemiology of Klebsiella bacteremic isolates and implications for vaccine development. J Clin Microbiol. 1986; 23:687-90.

34. Jones CL, Clancy M, Honnold C, Singh S, Snesrud E, Onmus-Leone F, et al. Fatal outbreak of an emerging clone of extensively drug-resistant Acinetobacter baumannii with enhanced virulence. Clin Infect Dis. 2015;61:145-54. https://doi.org/10.1093/cid/civ225

35. Melin M, Trzciński K, Meri S, Käyhty H, Väkeväinen M. The capsular serotype of Streptococcus pneumoniae is more important than the genetic background for resistance to complement. Infect Immun. 2010;78:5262-70. https:// doi.org/10.1128/IAI.00740-10

36. Rukke HV, Kalluru RS, Repnik U, Gerlini A, José RJ, Periselneris J, et al. Protective role of the capsule and impact of serotype 4 switching on Streptococcus mitis. Infect Immun. 2014;82:3790-801. https:/ / doi.org/10.1128/IAI.01840-14

37. Struve C, Roe CC, Stegger M, Stahlhut SG, Hansen DS, Engelthaler DM, et al. Mapping the evolution of hypervirulent Klebsiella pneumoniae. MBio. 2015;6:e00630. https://doi.org/10.1128/mBio.00630-15

38. Hsu C-R, Lin T-L, Chen Y-C, Chou H-C, Wang J-T. The role of Klebsiella pneumoniae rmpA in capsular polysaccharide synthesis and virulence revisited. Microbiology. 2011;157:3446-57. https://doi.org/10.1099/mic.0.050336-0

Address for correspondence: Yonghong Xiao, First Affiliated Hospital of Zhejiang University, Qingchun Rd 79, Hangzhou, Zhejiang 330003, China; email: xiaoyonghong@zju.edu.cn 